\title{
Primary malignant fibrous histiocytoma of the colon: A case report and review of the literature
}

\author{
WUYANG JI ${ }^{1}$, MINER ZHONG ${ }^{1}$, YAN YOU ${ }^{2}, \mathrm{KE} \mathrm{HU}^{3}$ and BIN WU ${ }^{1}$ \\ Departments of ${ }^{1}$ General Surgery, ${ }^{2}$ Pathology and ${ }^{3}$ Radiotherapy, Peking Union Medical College Hospital, \\ Peking Union Medical College, Chinese Academy of Medical Sciences, Beijing 100730, P.R. China
}

Received November 9, 2015; Accepted March 24, 2016

DOI: $10.3892 / \mathrm{mco} .2016 .849$

\begin{abstract}
A 68-year-old woman was admitted to the Peking Union Medical College Hospital with a 6-week history of fever of undetermined origin. The computed tomography scan revealed a space-occupying lesion in the ascending colon and colonic endoscopy revealed a protrusive lesion in the initial segment of the ascending colon, occupying $3 / 5$ of the lumen. Pathological examination of a biopsy specimen revealed malignant fibrous histiocytoma (MFH). The patient then underwent a laparotomy for right hemicolectomy and en bloc lymph node dissection. The histopathological examination confirmed the diagnosis of MFH and administration of adjuvant radiotherapy was decided. MFH is a high-grade sarcoma that rarely arises in the alimentary tract. To the best of our knowledge, there has only been one case of primary colorectal MFH treated by radiotherapy recorded to date, which makes our case the second trial. We herein present a case of MFH in the ascending colon and a review of previous case reports of patients presenting with this unusual type of MFH.
\end{abstract}

\section{Introduction}

Malignant fibrous histiocytoma (MFH) is a high-grade sarcoma that usually involves the soft tissue of the extremities and the retroperitoneum, but seldom arises in the alimentary tract. Since the late 1970 s, $<30$ cases of colorectal MFH have been documented, whereas little is known on its histogenesis and optimal treatment. MFH is a type of histiocytoma and the most common soft tissue sarcoma of late adult life (5th to 7 th decades), accounting for $21 \%$ of all soft-tissue sarcomas in a Japanese study of 130 cases (1). The major locations for this tumor are the lower (49\%) and upper extremities (19\%), while tumors of the retroperitoneum and abdominal

Correspondence to: Professor Bin Wu, Department of General Surgery, Peking Union Medical College Hospital, Peking Union Medical College, Chinese Academy of Medical Sciences, 1 ShuaiFuYuan, Beijing 100730, P.R. China

E-mail: wubin0279@hotmail.com

Key words: malignant fibrous histiocytoma, sarcoma, colon, operation, radiotherapy cavity account for $16 \%$, and those of head and neck account for $9 \%$ of the cases (2). We herein present a case of MFH of the ascending colon and discuss the characteristics of this tumor with reference to the literature.

\section{Case report}

A 68-year-old woman was admitted to the Peking Union Medical College Hospital in 2009 with a 6-week history of fever of undetermined origin (FUO). There was no recent history of cough, runny nose, pharyngalgia, vomiting or diarrhea. Antibiotic therapy proved ineffective and the patient was repeatedly administered non-steroidal anti-inflammatory drugs to control the temperature. She also exhibited increased nocturia over the past 15 years and a weight loss of $10 \mathrm{~kg}$ over the 6-week period of FUO. The patient was of average build and fairly well-nourished. On physical examination, there were no remarkable findings during abdominal palpation; however, $0.5-\mathrm{cm}$ lymph nodes in the right neck and axilla were palpable, without tenderness. Furthermore, the liver border was palpable one finger width below the costal margin. The past medical history and family history were unremarkable.

Laboratory tests revealed a white blood cell count of $8.69 \times 10^{9} / 1$ (neutrophils, 85.5\%), hemoglobin concentration of $75 \mathrm{~g} / 1$, platelet count of $552 \times 10^{9} / 1$, C-reactive protein (CRP) level of $119 \mathrm{mg} / \mathrm{l}$ and erythrocyte sedimentation rate $(\mathrm{ESR})>140 \mathrm{~mm} / \mathrm{h}$. The stool occult blood test was positive and urinalysis was negative. The tumor markers carcinoembryonic antigen and carbohydrate antigen 19-9 were within normal limits. Abdominal ultrasonography indicated a hypoechoic mass measuring $5.0 \times 4.4 \mathrm{~cm}$. Abdominal and pelvic computed tomography (CT) scans revealed a space-occupying lesion in the ascending colon (Fig. 1). Following a barium enema, filling defects of the ascending colon were identified. Colonic endoscopy revealed edema of the ileocecal valve and a protrusive lesion in the initial segment of the ascending colon, occupying $3 / 5$ of the lumen, with necrosis and a nodular surface. The mucosa appeared fragile, exhibiting spontaneous and contact bleeding. Pathological examination of a biopsy specimen indicated MFH and a preoperative diagnosis of a malignant tumor of the ascending colon was made.

The patient underwent laparotomy, which revealed a large mass sized $8.0 \times 6.0 \mathrm{~cm}$ in the ascending colon, accompanied by edema and local infiltration of the retroperitoneum, with 
unclear boundaries. Distal metastases to the retroperitoneal space were not identified. A few enlarged lymph nodes were detected the pericolic adipose tissue, but no invasion to the adjacent organs or metastasis to the liver was identified. We performed a right hemicolectomy and en bloc lymph node dissection.

Macroscopically, the surgical specimen included a well-circumscribed cauliflower-like tumor in the ascending colon, which was fragile and bled easily, and protruded both intraluminally and extramurally (Fig. 2). Histopathological examination revealed that the tumor was pleomorphic and composed of fibroblasts, myofibroblasts and histiocyte-like cells arranged in a storiform pattern (Fig. 3A). There was an abundant of bizarre tumor giant cells admixed with rounded histiocyte-like and chronic inflammatory cells (Fig. 3B). There was no lymph node involvement or invasion of lymphatic or blood vessels. However, the mass resected from the retroperitoneal space revealed malignant tumor cell invasion. No residual tumor cells were identified on the surgical margin. Immunohistochemical staining were positive for vimentin (Fig. 3C) and negative for S-100, CD68, CD117, anti-smooth muscle antibody and lysozyme. The final histopathological diagnosis was MFH of the ascending colon.

Considering this patient presented with retroperitoneal invasion, we decided to administer postoperative radiotherapy. However, there is no clear guideline regarding adjuvant radiotherapy of MFH in the colon. Following discussion, we decided to administer radiotherapy for 5 weeks $(50 \mathrm{~Gy} / 25$ fractions, $2 \mathrm{~Gy} / \mathrm{fr}, 5$ times per week). The patient developed grade 1 digestive tract reactions, without myelosuppression. After 3 years, the patient developed local recurrence and eventually succumbed to the disease after a further 2 years.

\section{Discussion}

MFH was first described as malignant histiocytoma and fibrous xanthoma by Ozello et al in 1963 (3), and as malignant fibrous xanthoma by the same group in the following year. In 1978, Weiss and Enzinger recommended that MFH be subcategorized into four variant histological subtypes, namely myxoid, storiform-pleomorphic, giant-cell and inflammatory (4). In the most recent WHO classification of soft tissue tumors in 2013 , a new category of 'undifferentiated/unclassified sarcoma' has been created and MFH falls into this category (5).

The cause and origin of MFH have not been fully elucidated; however, it has been recognized as a complication following irradiation, chronic postoperative repair, trauma, surgical incisions or burn scars (6). The main symptoms of colonic MFH are abdominal pain, fever, a palpable mass, blood in the stool, diarrhea and anorexia, resulting from the large mass. In addition, patients may develop other non-specific symptoms, including weight loss and increased intra-abdominal pressure (4). When compared with more common types of colorectal cancer, fewer patients had constipation, but $25 \%$ had fever. Blood analysis revealed leukocytosis, with elevated ESR and CRP levels, particularly in patients with an inflammatory response (7). In our case, the patient had fever persisting for 6 weeks, with elevated ESR and CRP levels. In addition to those symptoms and the laboratory findings, immunohistochemical examination is necessary, combined with changes in
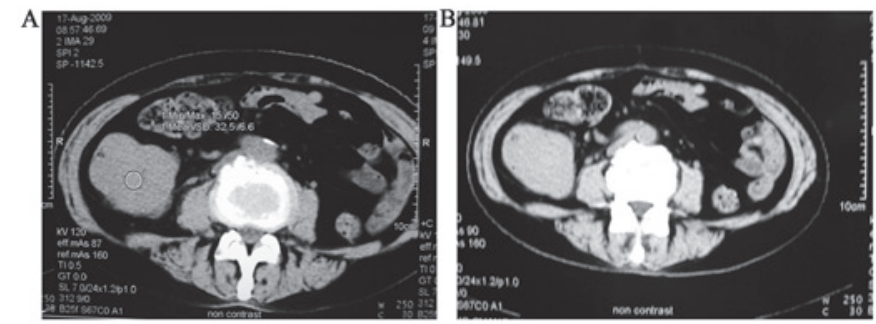

Figure 1. Abdominal computed tomography scan. (A) Non-contrast phase: A large mass with soft-tissue density was identified in the ascending colon; (B) portal venous phase: The mass measured $6.2 \times 5.2 \mathrm{~cm}$, with heterogeneous density.

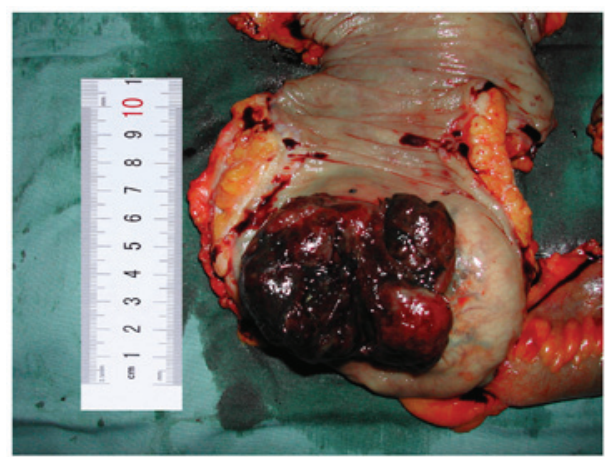

Figure 2. Surgical specimen showing a well-circumscribed tumor in the ascending colon. Macroscopically, the mass was solid, firm, lobulated (cauliflower-like appearance), sized $6.5 \times 7.7 \times 4.0 \mathrm{~cm}$ and reddish black in color.

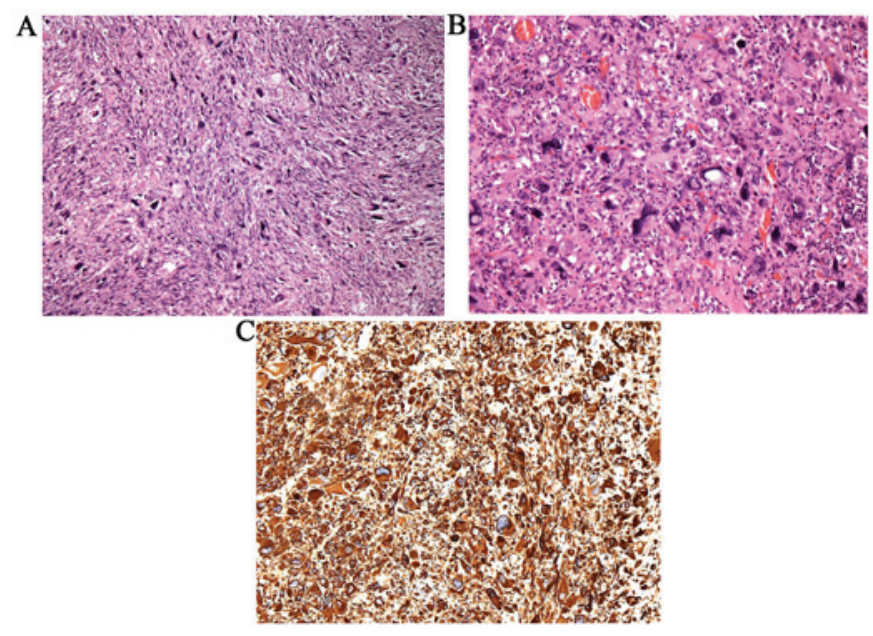

Figure 3. Pleomorphic malignant fibrous histiocytoma. (A) The tumor exhibited marked cytological and nuclear pleomorphism, with a storiform growth pattern [hematoxylin and eosin staining (H\&E); magnification, x100]. (B) On high-power magnification, an abundance of bizarre tumor giant cells admixed with rounded histiocyte-like and chronic inflammatory cells was observed (H\&E; magnification, $\mathrm{x} 400$ ). (C) On immunohistochemical examination, the tumor cells exhibited marked positivity for vimentin (magnification, $\mathrm{x} 200$ ).

nomenclature and more stringent histopathological criteria, in order to accurately diagnose this neoplasm (8).

The treatment for colonic MFH is early and complete surgical excision, combined if possible with en bloc resection of the regional lymph nodes, with or without radiotherapy. Considering the high recurrence rate and poor prognosis, in patients exhibiting large tumor size, high grade, or metastatic 
disease, adjuvant chemotherapy or radiotherapy may be recommended. However, there remains controversy and different trial results are inconsistent. For metastasis and relapse, the major determinants are histology (myxoid) and tumor size. Myxoid tumors exhibit lower metastatic propensity (10-year metastatic rate, $13 \%$ ) compared to non-myxoid tumors (10-year metastatic rate, 40\%) (6). Thus, patients with myxoid tumors may not require systemic therapy, while patients with non-myxoid disease exceeding $5.0 \mathrm{~cm}$ are at a significant risk of developing metastases. The development of effective adjuvant treatment is a major research goal (9). An increasing number of reports indicate that adjuvant chemotherapy or radiation may improve prognosis.

In our patient, the tumor infiltrated the retroperitoneum. Although the surgical margin of resection was sufficient, we decided to administer radiotherapy. To the best of our knowledge, there has been only one other case of primary colorectal $\mathrm{MFH}$ recorded to date, in which the patient was treated with radiotherapy. Thus, our case represents the second trial assessing the radiotherapeutic effect. Our patient completed one 5-week radiotherapy cycle, and developed only grade 1 digestive tract reactions, without myelosuppression. However, she later developed local recurrence (3 years post-treatment), and eventually succumbed to the disease after a further 2 years.

As regards the histological and immunohistochemical staining results, MFH is composed of histiocytic as well as fibrous elements, often arranged in a storiform pattern and accompanied by pleomorphic giant cells, xanthoma cells and inflammatory cells (7). It was reported that MFH frequently expresses vimentin, actin, CD68, $\alpha$ 1-antitrypsin and $\alpha 1$-antichymotrypsin, and often laminin mRNA (10). Our case was diagnosed as storiform $\mathrm{MFH}$, which is the most common type.

Local recurrence is most commonly associated with MFH of the large intestine, as those tumor have a tendency to originate submucosally and spread radially through the muscle to infiltrate surrounding structures. The risk of local recurrence and distant metastasis correlates with the depth and size of the primary tumor (11). The overall 2- and 5-year survival rates for MFH are $\sim 60$ and $47 \%$, respectively (4). The prognosis associated with colonic MFH is unclear, but most of the reports suggest unfavorable outcomes, due to the propensity of MFH to invade contiguous organs and to recur locally (12). The lung is the most frequent (82\%) metastatic site of MFHs (7). Complete surgical excision in the early stages is crucial for improving the prognosis of $\mathrm{MFH}$, although the role of adjuvant therapy following surgery remains unclear. Long-term follow-up with regular chest X-rays and CT scans of the abdomen to detect tumor recurrence, metastasis and lymph node involvement are mandatory (13). The clinical behavior of colonic MFH requires further investigation in a larger population with a longer follow-up.

\section{References}

1. Enjoji M, Hashimoto H, Tsuneyoshi M and Iwasaki H: Malignant fibrous histiocytoma. A clinicopathologic study of 130 cases. Acta Pathol Jpn 30: 727-741, 1980.

2. Cormier JN and Pollock RE: Soft tissue sarcomas. CA Cancer J Clin 54: 94-109, 2004

3. Ozello L, Stout AP and Murray MR: Cultural characteristics of malignant histiocytoma and fibrous xanthomas. Cancer 16: 331-344, 1963.

4. Weiss SW and Enzinger FM: Malignant fibrous histiocytoma: An analysis of 200 cases. Cancer 41: 2250-2266, 1978.

5. Jo VY and Fletcher CD: WHO classification of soft tissue tumors: An update based on the 2013 (4th) edition. Pathology 46: 95-104, 2014.

6. Fu DL, Yang F, Maskay A, Long J, Jin C, Yu XJ, Xu J, Zhou ZW and Ni QX: Primary intestinal malignant fibrous histiocytoma: Two case reports. World J Gastroenterol 13: 1299-1302, 2007.

7. Okubo H, Ozeki K, Tanaka T, Matsuo T and Mochinaga N: Primary malignant fibrous histiocytoma of the ascending colon: Report of a case. Surg Today 35: 323-327, 2005.

8. Daugaard S: Current soft-tissue sarcoma classifications. Eur J Cancer 40: 543-548, 2004.

9. Zagars GK, Mullen JR and Pollack A: Malignant fibrous histiocytoma: Outcome and prognostic factors following conservation surgery and radiotherapy. Int J Radiat Oncol Biol Phys 34: 983-994, 1996.

10. Soini $\mathrm{Y}$ and Autio-Harmainen H: Tumor cells of malignant fibrous histiocytoma express mRNA for laminine. Am J Pathol 139: 1061-1068, 1991.

11. Dong J, An W, Ma W, Guo X, Gao Y, Liu C and Li Y: Primary hepatic malignant fibrous histiocy toma mimicking hepatocellular carcinoma: A report of two cases. Oncol Lett 8: 2150-2154, 2014.

12. Chen HC, Chen CJ, Jeng CM and Yang CM: Malignant fibrous histiocytoma presenting as hemoperitoneum mimicking hepatocellular carcinoma rupture. World J Gastroenterol 13: 6441-6443, 2007.

13. Atmatzidis KS, Pavlidis TE, Galanis IN, Papaziogas BT and Papaziogas TB: Malignant fibrous histiocytoma of the abdominal cavity: Report of a case. Surg Today 33: 794-796, 2003. 